\title{
AMENDMENTS
}

\section{Publisher Correction: Learning human-environment interactions using conformal tactile textiles}

Yiyue Luo, Yunzhu Li (D), Pratyusha Sharma, Wan Shou D, Kui Wu, Michael Foshey, Beichen Li, Tomás Palacios, Antonio Torralba and Wojciech Matusik

Correction to: Nature Electronics https://doi.org/10.1038/s41928-021-00558-0, published online 24 March 2021.

In the version of this Article originally published, in Fig. 4c a technical issue resulted in the bottom photograph in the left column appearing incorrectly as a black square; this has now been corrected in all versions of the Article.

Published online: 1 April 2021

https://doi.org/10.1038/s41928-021-00572-2

(C) The Author(s), under exclusive licence to Springer Nature Limited 2021 